\title{
About the impact of the materials properties in the catastrophic degradation of high power GaAs based laser diodes
}

\author{
J.Souto, J.L.Pura, A.Torres, J.Jiménez \\ GdS Optronlab, Ed. i+d, Paseo de Belén, 11, Universidad de Valladolid, 47011 Valladolid, \\ Spain
}

\begin{abstract}
The catastrophic degradation of high power lasers depends on both external factors, associated with the technological processes followed to fabricate the laser, and also on intrinsic aspects related to the materials forming the laser structure, more specifically the active zone composed by the QW, guide layers and claddings. The materials properties: optical, thermal and mechanical, play a paramount role in the degradation of the laser. We analyse here how these properties have an impact on the mechanisms responsible for the catastrophic degradation.
\end{abstract}

Keywords: High Power laser diodes, catastrophic degradation, cathodoluminescence (CL), thermal stress, thermal conductivity

\section{INTRODUCTION}

The catastrophic optical degradation (COD) is the sudden drop of the optical power output of laser diodes after hours of regular operation [1]. It is well known that the threshold for COD has been pushed up by the improvement of the epitaxial growth, passivation, laser structure and packaging; however, the increasing demand of optical power renders the COD a critical challenge for applications requiring high power and reliability. This degradation is described in terms of a thermal runaway process, in which a positive feedback produces the melting of the active zone of the laser [2].

Dark line defects (DLDs) along the laser cavity typically appear as the products of the COD [3]. The occurrence of these DLDs can be detected at the front facet, but as the passivation and facet structures are improved the DLDs are triggered inside the cavity. The DLDs are aligned along the laser cavity, which suggests that their propagation is guided by the laser field, instead of being related to a specific crystallographic direction. The energy necessary for the propagation of the DLDs is supplied by the absorption of the laser radiation in regions overheated with respect to the QW operation temperature in the unperturbed zones of the laser cavity. Therefore, the COD appears as a local generation of defects that once triggered self-propagate leading to the laser end of life. The local temperature increase, once a critical temperature is reached [3], can be considered as the onset of the degradation process, since it induces the generation of dislocations in the active zone of the laser due to the thermal stresses locally generated. Therefore, one has to consider the impact of the optical (optical absorption), thermal (thermal conductivity), and mechanical (yield strength) properties of the laser structure on the processes leading to COD. The laser structure is formed by a stack of layers of different compositions and thicknesses, which affect all of those properties. We discuss herein about the impact of the materials properties on the laser degradation, which of them are detrimental to the laser reliability and which are responsible of the laser endurance. 


\section{DARK LINE DEFECTS}

The DLDs are the typical products of the laser degradation both in broad emitter and single mode lasers. The DLDs are observed by cathodoluminescence (CL) and electron beam induced current (EBIC), both starting at the front facet and inside the cavity. The DLDs appear in the CL images as dark contrasted lines, Fig. 1; they propagate along the optical path. Some DLDs spread out laterally following specific crystallographic directions, but this are secondary products of the main DLD aligned along the optical field [4]. Note that in broad emitter lasers the DLDs are a little more complex than in single narrow ridge monomode lasers, because of the existence of circulating modes, Fig.1.
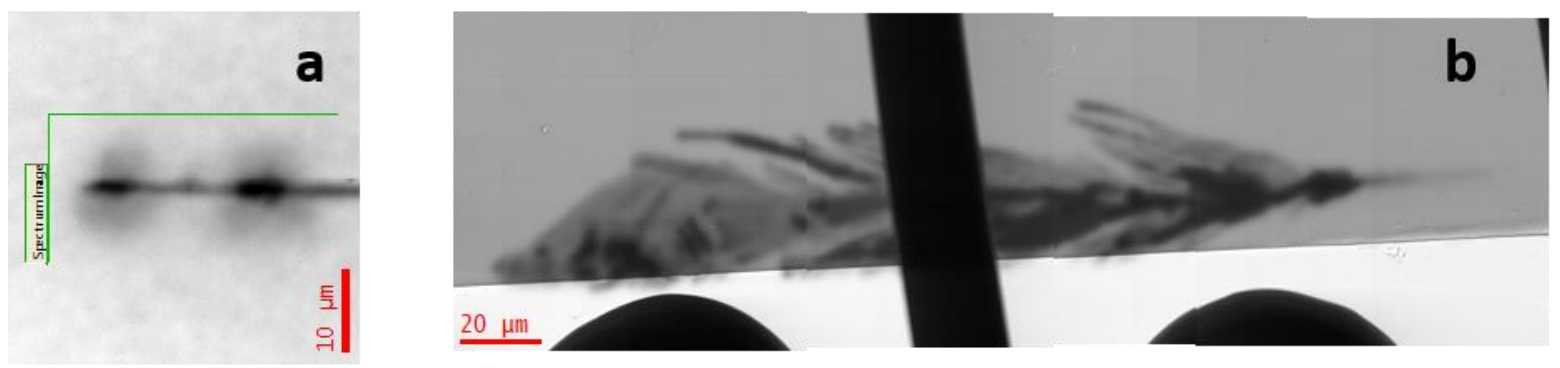

Fig.1. DLDs, along the ridge of a single monomode $980 \mathrm{~nm}$ laser, CL image (a), DLDs in a broad emitter 980 nm laser, EBIC image (b)

The DLD is localized in the QW, though in the most degraded zones it extends to the waveguide layers, Fig.2. It is usually admitted that both temperature and stress play a major role in the laser degradation and the subsequent DLD formation. The DLDs are usually associated with the formation of dislocations. In previous works we have developed a thermo-mechanical model accounting for the formation of dislocations in the active zone of the lasers [5]. In this model a local temperature increase induces thermal stresses, which are responsible for the plastic deformation that marks the beginning of the DLDs formation. However, it is well known that different lasers, both in terms of the composition of the active layer as well as in terms of the structure, degrade in different ways, e.g. double hetero-structure lasers have lower power damage threshold than QW lasers; also, the degradation power threshold in strained QW lasers is higher than in lattice matched QWs [2]. The end of lasing is observed as the guide layers are degraded, i.e. once the dislocations generated in the QW are propagated inside the guide layers. This behavior and the degradation mechanism previously described suggests that there are physical properties of the laser structure that play a paramount role in the degradation of the lasers.

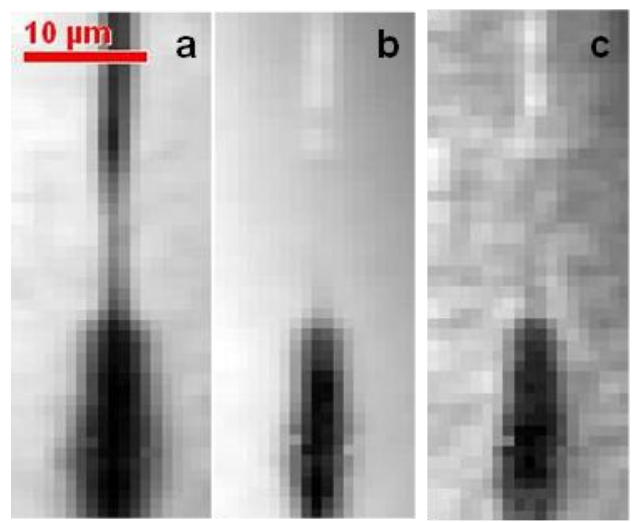

Fig.2. CL image of the DLD along the ridge of a monomode laser (a) QW emission, (b) n-guide, (c) p-guide 


\section{PHYSICAL PROPERTIES GOVERNING THE LASER DEGRADATION}

As mentioned above, the formation of dislocations is the consequence of the thermal stresses induced by local heating; therefore, thermal and mechanical properties of the laser structure play a major role in the laser degradation process. A second point of interest concerns the optical absorption. Under normal operation the QW and the guides are transparent to the laser radiation. When the temperature is locally enhanced the QW becomes an absorbing medium at the locally heated region. Note that the laser radiation is being generated in the regions of the QW remaining at the operating temperature. The guide layers remain transparent, intrinsic absorption by the guides can only occur at very high temperature. However, the calculated temperature at the guide layers is significantly lower than the QW temperature, which rules out intrinsic laser absorption by the guide layers [6].

The local absorption of the laser radiation feedbacks the heat source in the QW, and the heat dissipation is governed by the thermal conductivity of the laser structure, which determines the temperature distribution around the hot spot. Usually, one considers bulk figures; however, in a multilayer structure with very thin layers, a dramatic decrease of the thermal conductivity takes place because of the reduced thickness of the layers, which is especially relevant for the QW, which is only a few nanometers thick, well below the Casimir limit. Furthermore, one has to consider the interfaces, which introduce thermal resistance; depending on the quality of the interfaces the phonon scattering can be specular or diffusive. At the actual state of the art of the epitaxial layers one can assume that initially the interfaces have good quality.

Bearing in mind the typical power emission of this type of devices, one can assume that the absorbed laser power densities for continuous wave operation may lie between 0 and $10-12 \mathrm{MW} / \mathrm{cm}^{2}$ [7], which means that the heat power locally generated lies within such an interval of power densities. By solving the heat transport equation one can estimate the temperature distribution around the heat source. At this point the thermal conductivity plays a capital role, because the temperature distribution will be strongly dependent on the local thermal resistance. This will be determined by the phonon mismatch between the different layers, mainly between the QW and the barrier layers, and also by the nature of the interfaces. The results plotted in Fig. 3 represent the local QW temperature as a function of the absorbed laser power density for different $\kappa e f f / \kappa b u l k$ ratios, where кeff is the effective thermal conductivity of the lasing layer, in which the contributions of the QW thickness and the thermal resistance of the interfaces are considered, and kbulk is the thermal conductivity for bulk material. The temperature of the hot spot in the QW increases with the laser power density absorbed. This dependence is initially nearly linear, and becomes slightly superlinear for increasing power densities. This behavior is strongly constrained by the keff/kbulk ratio, the temperature is sharply increased for decreasing

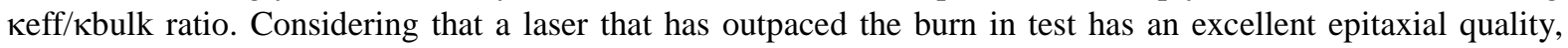
free of extended defects, one can assume that the кeff/kbulk ratio must be relatively high at the beginning of the process; therefore, a sudden degradation under the range of laser power densities at which these lasers operate can hardly be expected. However, one has to take account of the evolution of the laser structure during the laser operation. In previous papers we have developed thermo-mechanical models accounting for the degradation process. The thermal stresses induced by the temperature gradient associated with the hot spot would be responsible for the formation of dislocations. In the presence of defects the thermal conductivity is further decreased [7], which would lead to a steeper temperature increase, which becomes strongly superlinear. In fact, the result of a reduced thermal conductivity is a high local concentration of energy at the hot spot, leading to the thermal micro-explosion [8], or micro-ignition [9] that characterizes the thermal runaway process. The thermal conductivity is therefore a physical parameter playing a critical role in the laser degradation.

As we have mentioned, thermal stresses are responsible for the formation of the dislocations triggering the thermal runaway process. In this way, the mechanical endurance of the laser structure is a critical issue for the resistance to degradation. The thermal stresses can be evaluated by solving the corresponding elastic equations. The main thermal stresses are concentrated on the QW, where the dislocations are formed once a critical stress (yield strength) is reached. Therefore, the mechanical endurance of the QW is crucial to the laser reliability. Usually, one considers the plastic deformation values reported for bulk materials, without keeping in mind the low dimensionality of the layers conforming the laser structure, very specially the QW. There is a huge amount of work on the mechanical strength of low dimensionality structures. Remarkable high yield strengths have been reported for different materials at the submicron scale [10]. All this has been summarized in different 
empirical approaches: the Hall-Petch or the modified confined layer slip (CLS) models. These models determine the yield strength of nanostructured materials in terms of the lowest dimension of the system (h), the yield strength increasing for decreasing $h$; this dependence is fixed as $h^{-1 / 2}$ for Hall-Petch, and $\ln (h) / h$ for the modified CLS models, respectively [10], in the case of the laser structure h corresponds to the QW thickness.

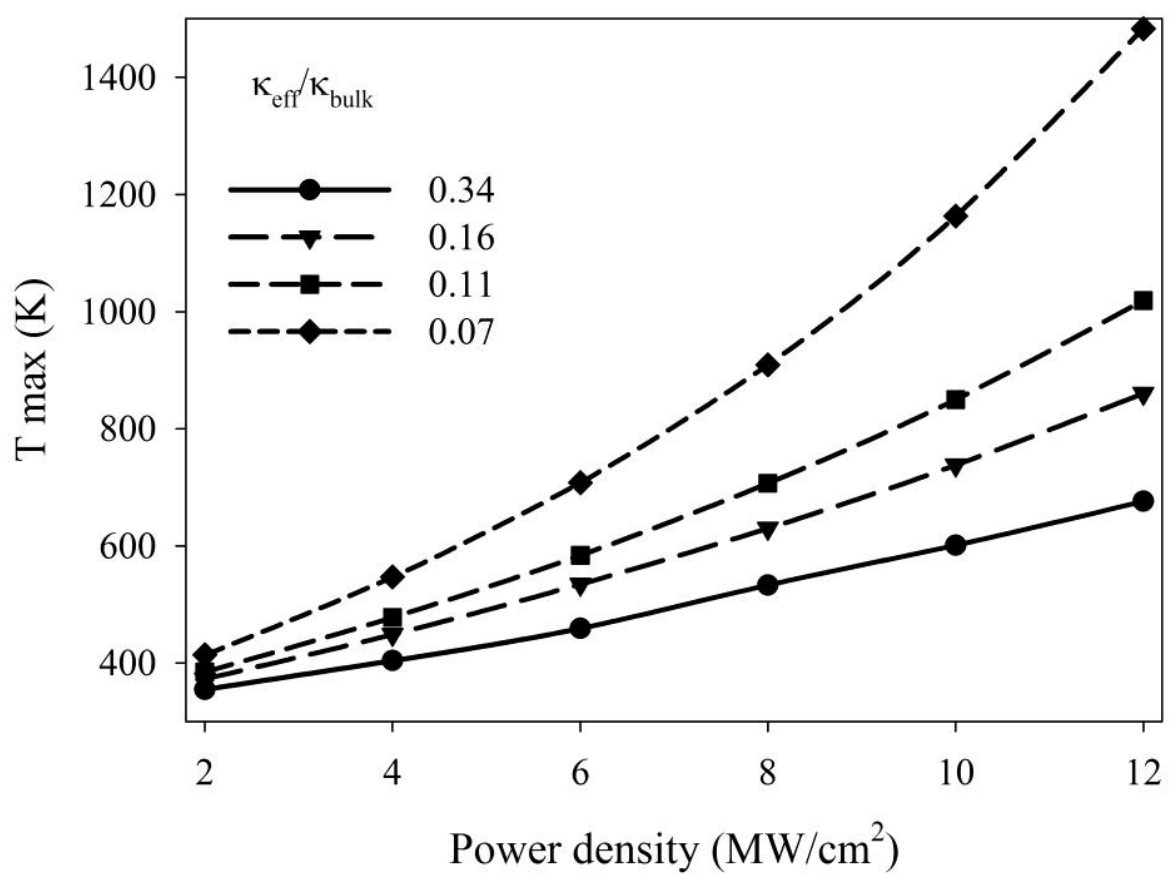

Fig. 3. Maximum temperature in the laser diode in terms of the dissipated power density in the QW for different

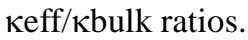

Although a considerable body of experimental results is available for systems based on metals, a limited amount of information exists for the mechanical behavior of GaAs in the micro- and nanoscales [11-13]. As an example, a drastic departure from bulk values was observed for GaAs nanowires, for which an enhancement of Young's modulus in the nanometer regime was reported [13]. According to these works, the behavior of GaAs would follow similar trends to those of metal-based systems, which would account for a significant endurance of the QWs, which will present a much higher threshold for plastic deformation than what is usually assumed. The plot of the shear stress (Tresca) vs the QW temperature for different power densities and thermal conductivity ratios is shown in Fig. 4; interestingly all the points are aligned. The threshold for plastic deformation is somewhere along this line, depending on the mechanical strength of the active layer: if one pushes the threshold along this line, more reliable lasers can be achieved. This can be done by the selection of the composition and thickness of the active layers. It is interesting to mention the strong interrelation between the temperature, which is basically determined by the thermal conductivity, and the absorbed laser power and the thermal stress. Therefore, thermal conductivity and mechanical strength are fundamental physical properties to be engineered for the preparation of robust laser diodes.

Finally, it is well known that strained QW lasers are more reliable than unstrained QW lasers. One has not convincing arguments about it, since it appears contradictory with the role of stress as an accelerator factor for the laser degradation. Here, we have to argue about the mechanism leading to the end of lasing in a degraded device. We have described about the formation of the DLDs, which are associated with the generation of dislocations at the QW due to the thermal stresses. However, degraded lasers show that damage is extended to the barrier layers. In fact, the local damage of the QW does not by itself destroy the laser cavity, it only reduces the laser gain. Instead of this, the destruction of the laser cavity should be due to the degradation of the guide 
layers. It should be noted that the guide layers are transparent to the laser radiation. Though no intrinsic absorption of the laser radiation is to be expected, the guide layers can become non transparent when the dislocations generated in the QW by the above described mechanisms propagate inside them. Interestingly, it is this mechanism that is inhibited, or at least delayed, in strained QW. There is empiric evidence of this effect in laser structures: in CL images of DLDs in strained QW lasers one can observe dislocations mainly localized in the QW [3, 14]. Moreover, a significant body of research about the propagation of dislocations in strained systems exists for metals, showing that strain inhibits this mechanism [10, 15].

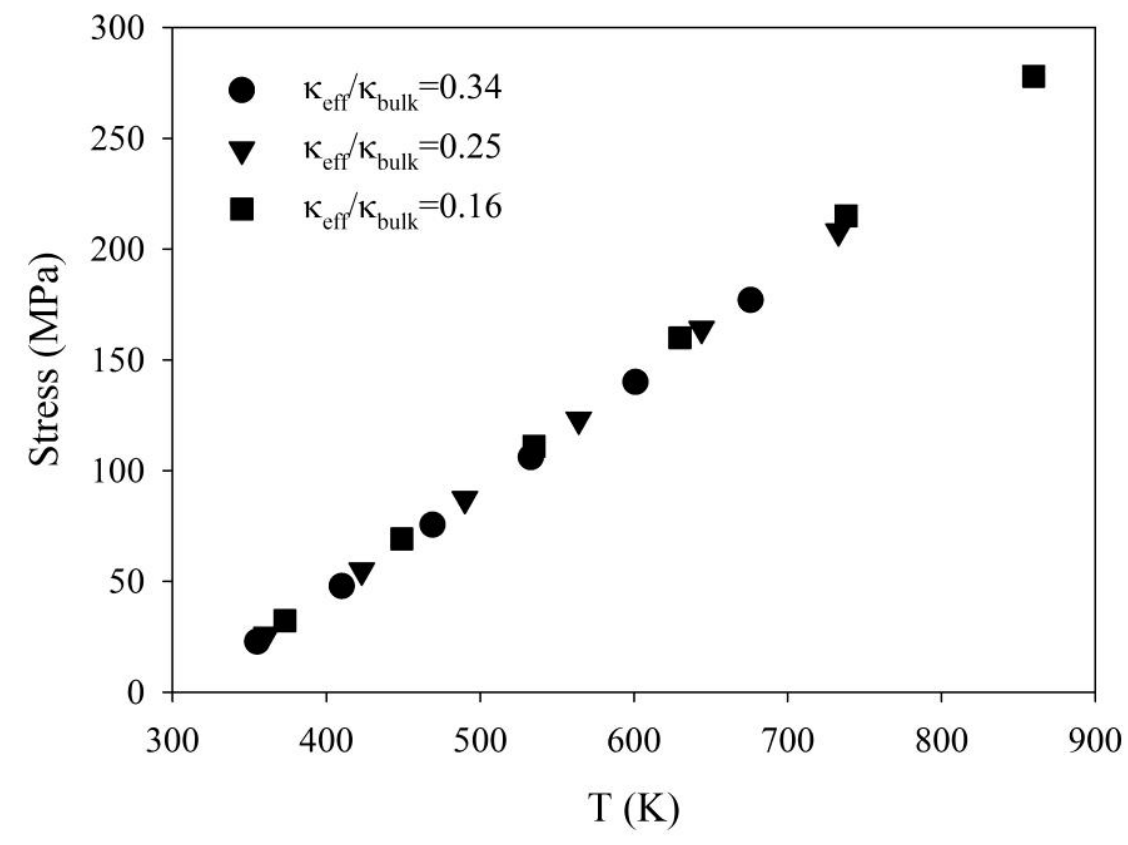

Fig. 4. Maximum shear stress versus QW peak temperature for different thermal conductivity ratios and absorbed power densities (between 2 and $12 \mathrm{MW} / \mathrm{cm} 2$, with a $2 \mathrm{MW} / \mathrm{cm}^{2}$ step size).

\section{CONCLUSION}

In summary, the materials properties of the laser structure, related to the composition of the layers, and the structure of the multilayer system forming the active parts of the laser play a major role in the laser reliability. Thermal and mechanical properties appear as critical issues for the laser endurance; the engineering of these properties should allow the improvement of the laser diode reliability.

\section{ACKNOWLEDGMENTS}

This work was funded by the Spanish Government (Grant: ENE2014-56069-C4-4-R) and Junta de Castilla y León (VA293U13 and VA081U16 (003)).

\section{REFERENCES}

[1] Jiménez J. “ Laser diode reliability: Crystal defects and degradation modes” Comptes Rendus Phys. 4 66373 (2003) 
[2] Eliseev, P. G. "Optical strength of semiconductor laser materials" Prog. Quantum Electron. 20, 1-82 (1996)

[3] Hortelano V, Anaya J, Souto J, Jiménez J, Perinet J and Laruelle F "Defect signatures in degraded high power laser diodes" Microelectron. Reliab. 53 1501-5 (2013)

[4] Hempel M, Tomm J W, Hortelano V, Michel N, Jiménez J, Krakowski M and Elsaesser T. "Timeresolved reconstruction of defect creation sequences in diode lasers" Laser Photon. Rev. 6 L15-9 (2012)

[5] Martín-Martín, A., Avella, M., Iñiguez, M. P., Jiménez, J., Oudart, M. and Nagle, J. "Thermomechanical model for the plastic deformation in high power laser diodes during operation" J. Appl. Phys. 106, 73105 (2009).

[6] Souto J, Pura J L and Jiménez J. "About the physical meaning of the critical temperature for catastrophic optical damage in high power quantum well laser diodes" Laser Phys. Lett. 1325005 (2016)

[7] Hopkins P E "Thermal transport across solid interfaces with nanoscale imperfections: Effects of roughness, disorder, dislocations, and bonding on thermal boundary conductance" ISRN Mech. Eng. 2013, 682586 (2013)

[8] Eliseev, P. G. "Degradation of injection lasers" J. Luminescence 7, 338-56 (1973)

[9] Tomm, J. W., Ziegler, M., Hempel, M. and Elsaesser, T. "Mechanisms and fast kinetics of the catastrophic optical damage (COD) in GaAs-based diode lasers" Laser Photon. Rev. 5, 422-41 (2011).

[10] Maharaj, D. and Bhushan, B. "Friction, wear and mechanical behavior of nano-objects on the nanoscale" Mater. Sci. Eng. R 95, 1-43 (2015).

[11] Michler, J., Wasmer, K., Meier, S., Östlund, F. and Leifer, K. "Plastic deformation of gallium arsenide micropillars under uniaxial compression at room temperature” Appl. Phys. Lett. 90, 88-91 (2007).

[12] Ghisleni, R., Liu, J., Raghavan, R., Brodard, P., Lugstein, A., Wasmer, K. and Michler, J. "In situ microRaman characterization of plasticity and fracture in GaAs” Philos. Mag. 91, 1286-92 (2011).

[13] Wang, Y. B., Wang, L. F., Joyce, H. J., Gao, Q., Liao, X. Z., Mai, Y. W., Tan, H. H., Zou, J., Ringer, S. P., Gao, H. J. and Jagadish, C. "Super deformability and young's modulus of GaAs nanowires" Adv. Mater. 23, 1356-60 (2011)

[14] Waters R.G., Bour D.P., Yellen S.L., and Ruggieri F. "Inhibited dark line defect formation in strained InGaAs/AlGaAs quantum well lasers" IEEE Photonics Technol. 2, 531-533 (1990)

[15] Goagland R.G., Kurtz R.J., and Henager Jr. C.H. "Slip resistance and the strength of metallic multilayer composites" Scr. Materialia 5, 775-779 (2004) 